
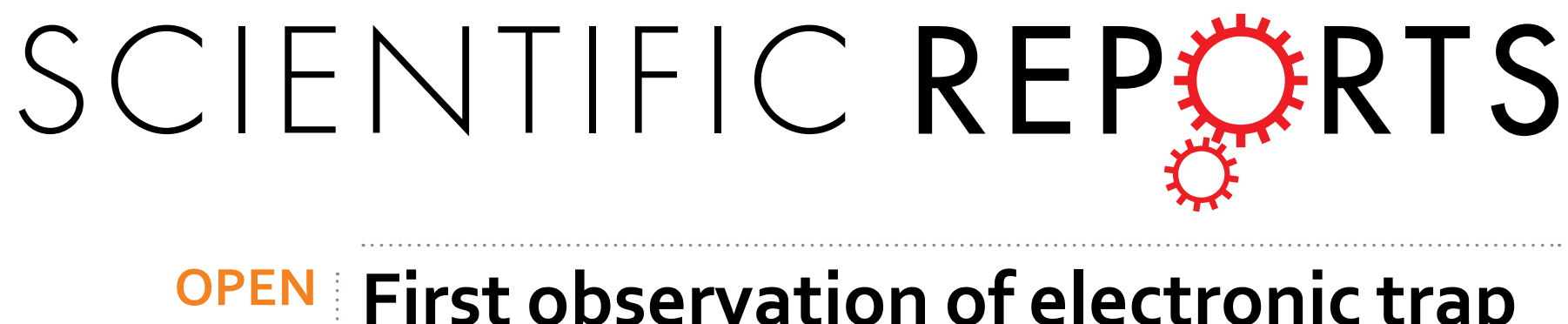

\title{
First observation of electronic trap levels in freestanding GaN crystals extracted from Si substrates by hydride vapour phase epitaxy
}

Received: 22 October 2018

Accepted: 27 April 2019

Published online: 09 May 2019

\author{
Moonsang Lee ${ }^{1}$, Chang Wan Ahn ${ }^{2}$, Thi Kim Oanh Vu², Hyun Uk Lee ${ }^{3}$, Eun Kyu Kim ${ }^{2}{ }^{2}$ \& \\ Sungsoo Park $^{4,5}$
}

The electronic deep level states of defects embedded in freestanding GaN crystals exfoliated from Si substrates by hydride vapour phase epitaxy (HVPE) is investigated for the first time, using deep level transient spectroscopy (DLTS). The electron traps are positioned $0.24 \mathrm{eV}$ (E1) and $1.06 \mathrm{eV}$ (E2) below the conduction band edge, respectively. The capture cross sections of E1 and E2 are evaluated to be $1.65 \times 10^{-17} \mathrm{~cm}^{2}$ and $1.76 \times 10^{-14} \mathrm{~cm}^{2}$ and the corresponding trap densities are $1.07 \times 10^{14} \mathrm{~cm}^{-3}$ and $2.19 \times 10^{15} \mathrm{~cm}^{-3}$, respectively. The DLTS signal and concentration of the electronic deep levels are independent of the filling pulse width, and the depth toward the bottom of the sample, evidenced by the fact that they are correlated to noninteracting point defects. Furthermore, Photoluminescence (PL) measurement shows green luminescence, suggesting that unidentified point defects or complex, which affect the optical characterisitics, exhibit. Despite the Si-based materials, the freestanding GaN exhibits deep level characteristics comparable to those of conventional freestanding $\mathrm{GaN}$, suggesting that it is a desirable material for use in the next generation optoelectronic devices with the large-scalibilityand low production costs.

Owing to their unique physical properties, gallium nitride $(\mathrm{GaN})$ and its related compounds are among the most promising materials for optoelectrical devices, such as laser diodes (LDs), ultra violet detectors, light-emitting diodes (LEDs), and high frequency and high power electronics ${ }^{1,2}$. To improve the performances of GaN-based devices, it is necessary to use freestanding (FS) GaN substrates. Because native bulk GaN does not exist, various growth methods, such as ammonothermal, high temperature high pressure (HTHP) growth, Na flux, and hydride vapour phase epitaxy (HVPE) have been employed to obtain FS-GaN to date ${ }^{3-6}$. Among these things, HVPE has good potential because it can provide a high growth rate and relatively high crystallinity ${ }^{7}$. Issues such as size limitations ( $<6$ inch diameter) and high production costs, however, have to be overcome for the commercialization of HVPE FS-GaN wafers ${ }^{8}$. Although the introduction of Si substrates as a supporting material could help resolve such issues, this process has been considered impossible owing to the generation of cracks in HVPE GaN layers and meltback effects ${ }^{9,10}$. Researchers have tried to obtain FS-GaN crystals from Si substrates using diverse approaches, such as an intermediate buffer layer, graded buffer layers, and $\mathrm{SiC}$ nano buffer layers ${ }^{11-13}$. However, there have not been any reports on the growth of FS-GaN crystals extracted from Si substrates. Recently, we demonstrated the growth of crack-free FS-GaN 2 inch in diameter and $400 \mu \mathrm{m}$ in thickness using in situ removal of the Si substrate by HVPE as well as its application in InGaN/GaN blue light emitting diodes (LEDs) ${ }^{14,15}$. However, the electrical properties of the defect states embedded in HVPE FS-GaN crystals detached from Si substrates have heretofore not been investigated. To appropriately utilize FS-GaN based on Si substrates, it is essential to explore and understand the origins and properties of defects in GaN layers peeled from Si substrates.

${ }^{1}$ Korea Basic Science Institute, Daejeon, 34133, Republic of Korea. ${ }^{2}$ Department of Physics, Quantum-Function Research Laboratory, Hanyang University, Seoul, 133-791, Republic of Korea. ${ }^{3}$ Advanced Nano-surface Research Group, Korea Basic Science Institute, Daejeon, 34133, Republic of Korea. ${ }^{4}$ Department of Science Education, Jeonju University, Jeonju, 303, Republic of Korea. ${ }^{5}$ Analytical Laboratory of Advanced Ferroelectric Crystals, Jeonju University, Jeonju, 303, Republic of Korea. Correspondence and requests for materials should be addressed to M.L. (email: Ims1015@kbsi.re.kr) or E.K.K. (email: ek-kim@hanyang.ac) or S.P. (email: krsspark@jj.ac.kr) 


\section{Si substrate}

\section{Buffer layer formation}

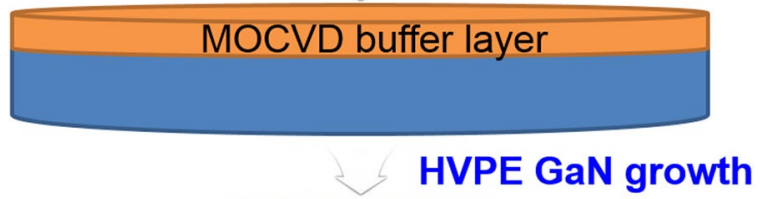

\section{HVPE GaN}

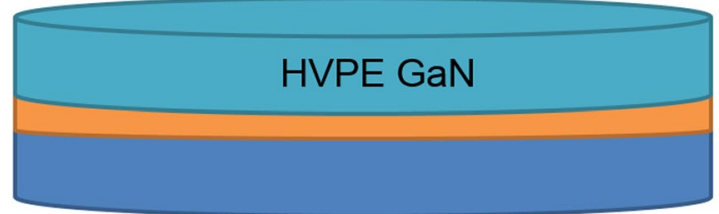

In-situ removal of Sub.

Si-based FS-GaN

\section{Contact formation}

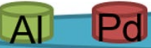

Si-based FS-GaN

Figure 1. Schematic illustration for the formation of Si-based FS-GaN Schottky diodes.

In this paper, we report the origin and electrical properties of electronic deep trap levels incorporated in the HVPE FS-GaN crystals extracted from Si substrates, using deep-level transient spectroscopy (DLTS). We expect that this study will shed light on the characterization of electronic states embedded in FS-GaN crystals peeled from Si substrates by HVPE, thus providing impetus to the performance optimization of optoelectronic devices that use FS-GaN crystals based on Si substrates.

\section{Experimental}

The FS-GaN crystals used in this study were grown from Si substrates, using an in-situ removal method by HVPE. The details of the growth process are described elsewhere ${ }^{15,16}$. A home-built vertical type HVPE system with an upstream $\mathrm{HCl}$ gas channel was used to achieve the FS-GaN crystal from a Si substrate. To form Si-based HVPE GaN layers, $\mathrm{AlN} / \mathrm{Al}_{0.4} \mathrm{Ga}_{0.6} \mathrm{~N}$ buffer layers were deposited on 2 inch $\mathrm{Si}$ (111) substrate, using metalorganic chemical vapour deposition (MOCVD). Next, HVPE GaN films were grown in atmosphere pressure with V/ III ratio of 20, followed by the in-situ etching of the substrate at $1273 \mathrm{~K}$. Finally, we could obtain the Si-based FS-GaN. To investigate the electrical characterization of the FS-GaN crystals, Schottky diodes were formed. First, 3-mm-diameter Al metal $(150 \mathrm{~nm})$ was deposited on one Ga-face of the FS-GaN to form Ohmic contacts, using an electron beam evaporator. Subsequently, 300- $\mu$ m diameter Pd Schottky contacts were formed on the other Ga-face surface by the electron beam evaporator, followed by rapid thermal annealing in ambient Ar at $823 \mathrm{~K}$ to improve the contact formation. The overall process to form S-based FS-GaN Schottky diodes is illustrated in Fig. 1. The measurements of deep levels in the FS-GaN were performed in a system developed in-house, using a $100 \mathrm{mV}$ signal at $1 \mathrm{MHz}$ within a temperature range of $100-420 \mathrm{~K}$. Moreover, the optical characteristics of the FS-GaN crystals were measured by photoluminescence (PL) analysis, which was excited using a He-Cd laser of $325 \mathrm{~nm}$ wavelength at room temperature.

\section{Results and Discussion}

A typical current-voltage (I-V) measurements of Pd/Si-based FS-GaN Schottky diode were employed at room temperature, as shown in Fig. 2(a). The diode exhibits a clear rectifying characteristics, implying that the Schottky junctions are well formed at metal/Si-based FS-GaN. Furthermore, the inset of Fig. 1(a) obviously represents that the forward and reverse leakage current increase exponentially with the bias, accompanying the shape of a thermionic field emission and trap-assisted tunneling ${ }^{17}$. This indicates that deep traps are present in the Si-based FS-GaN ${ }^{18}$. The detailed analysis of the $I-V$ curves will be elsewhere. To investigate the deep trap states incorporated in the Si-based FS-GaN crystals, DLTS measurements and their Arrhenius plots were conducted, respectively, as illustrated in Fig. 2(b,c). The DLTS spectrum was obtained at an emission rate $\left(\mathrm{e}_{\mathrm{n}}\right)$ of $0.90 \mathrm{~Hz}$ under an applied filling pulse width of $20 \mathrm{~ms}$, within a temperature range of 100-420 K. Two distinct deep level states, which are labelled as E1 and E2, can be seen.

The electronic states of E1 and E2 were centered at $0.24 \mathrm{eV}$ and $1.06 \mathrm{eV}$ below the conduction band edge, respectively. It is apparent that the trap E2 behaves as the dominant defect with a trap density of $2.19 \times 10^{15} / \mathrm{cm}^{3}$ 
(a)

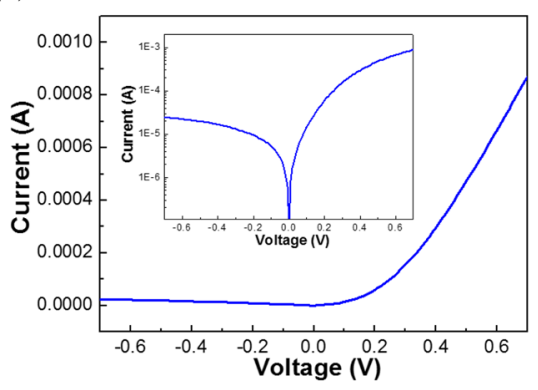

(b)

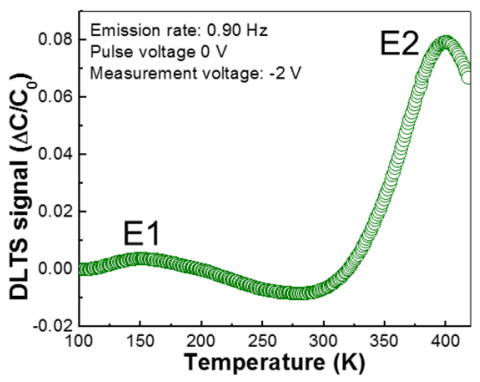

(c)

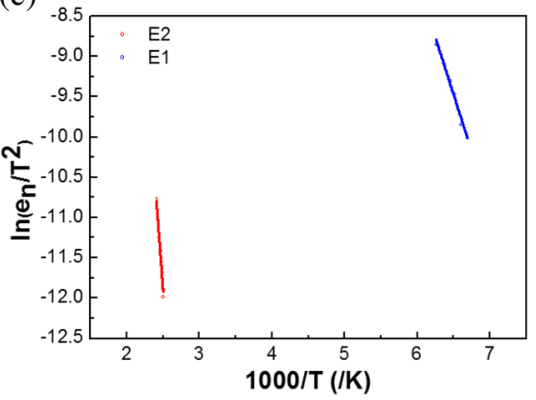

Figure 2. (a) $I-V$ characteristics of Pd/Si-based FS-GaN Schottky diode at room temperature. The inset depicts logarithmic plot of $I-V$ characteristics in Pd metal/Si-based Schottky diode. (b) DLTS spectrum of Si-based FS-GaN measured under a pulse voltage of $0 \mathrm{~V}$ and an applied voltage of $-2 \mathrm{~V}$ in the temperature range of $100-420 \mathrm{~K}$. (c) Arrhenius plot of the DLTS signal of the studied material.

\begin{tabular}{|l|l|l|l|}
\hline Defect & $\begin{array}{l}\text { Activation } \\
\text { energy }(\mathbf{e V})\end{array}$ & $\begin{array}{l}\text { Capture cross } \\
\text { section }\left(\mathrm{cm}^{2}\right)\end{array}$ & $\begin{array}{l}\text { Trap density } \\
\left(\mathrm{cm}^{-3}\right)\end{array}$ \\
\hline E1 & 0.24 & $1.65 \times 10^{-17}$ & $1.07 \times 10^{14}$ \\
\hline E2 & 1.06 & $1.76 \times 10^{-14}$ & $2.19 \times 10^{15}$ \\
\hline
\end{tabular}

Table 1. Defect parameters for the FS-GaN crystal extracted from a Si substrates by HVPE.

\begin{tabular}{|l|l|l|l|l|}
\hline Ref. & Activation energy $(\mathbf{e V})$ & $\begin{array}{l}\text { Capture cross section } \\
\left(\mathbf{c m}^{2}\right)\end{array}$ & Trap density & $\begin{array}{l}\text { Emission rate } \\
(\mathbf{H z})\end{array}$ \\
\hline \multirow{2}{*}{ This study } & 0.24 & $\begin{array}{l}1.65 \times 10^{-17} \\
1.76 \times 10^{-14}\end{array}$ & $\begin{array}{l}1.07 \times 10^{14} \\
2.19 \times 10^{15}\end{array}$ & 0.90 \\
\hline 19 & 1.06 & $10^{-12}-10^{-16}$ & $\sim 10^{12}-2.2 \times 10^{15}$ & 0.014 \\
\hline 20 & $0.25,0.563,0.65,0.69,1.40,1.55$ & $6.7 \times 10^{-14}-9.0 \times 10^{-16}$ & Mid-10 & $0.0125-1.25$ \\
\hline 21 & $0.25,0.35,0.59,0.66,1.0$ & $1.2 \times 10^{-15}-2.4 \times 10^{-15}$ & - & - \\
\hline 22 & $0.25,0.35,0.53,0.58,0.70$ & - & $\leq 10^{14}$ & - \\
\hline
\end{tabular}

Table 2. Summary of defect parameters in Si-based FS-GaN compared to other literatures.

and a capture cross section of $1.76 \times 10^{-14} / \mathrm{cm}^{2}$. A trap density and a capture cross section of E1 were estimated to be $1.07 \times 10^{14} / \mathrm{cm}^{3}$ and $1.65 \times 10^{-17} / \mathrm{cm}^{2}$, respectively, as shown in Table 1. Compared to the properties of deep levels in conventional HVPE FS-GaN crystals, the fingerprints of electronic states, which indicate a trap density and capture cross section, embedded in the Si-based FS-GaN are comparable ${ }^{19-22}$. (See Table 2) Furthermore, this is also comparable to the characteristics of the deep levels in the GaN layers grown using metalorganic chemical vapour deposition (MOCVD ${ }^{23-25}$. Notwithstanding HVPE with a high growth rate, which can cause a deterioration of crystal quality in the GaN layers, the properties of deep trap levels in the Si-based HVPE FS-GaN exhibit relatively nondegraded characteristics, compared to those of the preexisting GaN films. This strongly suggests that the Si-based FS-GaN crystals can realize large-scale high-performance opto-electronic devices without any electronic degradation.

To shed light on the origin of the trap states in the Si-based FS-GaN, we measured the deviation of the DLTS signals as a function of filling pulse time $\left(\mathrm{t}_{\mathrm{p}}\right)$, as depicted in Fig. 3. It is clear that the variations of the signals for the two deep levels are negligible. This implies that the two electronic deep levels originate from noninteracting defects, namely point ones. It is well known that the noninteracting defects depend exponentially on $\mathrm{t}_{\mathrm{p}}$. On the contrary, interacting defects related to dislocations and stacking faults, are proportional to $\ln (\mathrm{tp})$. Because the outlines of a DLTS signal as a function of $\ln (\mathrm{tp})$ in Fig. 2 are independent of $\ln \left(\mathrm{t}_{\mathrm{p}}\right)$, we can define the two defects as noninteracting point defects. As mentioned above, the E1 and E2 traps have been commonly observed in other GaN layers. $\mathrm{E} 1$ was considered as nitrogen or nitrogen vacancy $\left(\mathrm{V}_{\mathrm{N}}\right)$-related defects, denoted as $\mathrm{V}_{\mathrm{Ga}}-\mathrm{V}_{\mathrm{N}}$ pair ${ }^{24,26,27}$, and $\mathrm{E} 2$ is interpreted as nitrogen interstitial $\left(\mathrm{N}_{\mathrm{I}}\right)^{28}$ or line defects ${ }^{29,30}$.

Figure 4 illustrates the depth profiles of the trap densities of E1 and E2 and carrier concentration in the Si-based FS-GaN, obtained from the DLTS signal and capacitance-voltage profile, respectively. The depth from the surface of the Si-based FS-GaN became intense with increasing reverse bias voltage. As shown in Fig. 4(a), the behaviors of the two defect densities are similar, and these were almost unchanged throughout the sample depth. Slight increases in the lines at a depth of $200 \mathrm{~nm}$ were found. Surface defects due to electron-beam evaporation are responsible for such a feature ${ }^{31}$. Considering the nonlinear relationship between the DLTS signal and $\ln \left(\mathrm{t}_{\mathrm{p}}\right)$ and concentration depth profiles of E1 and E2, we concluded that these defects is inherited from the noninteracting point defects without any extended defect-related ones. Additionally, we implemented PL analysis of the studied materials, as presented in Fig. 4(b). Strong band-edge emission peaks of the Si-based FS-GaN crystals 


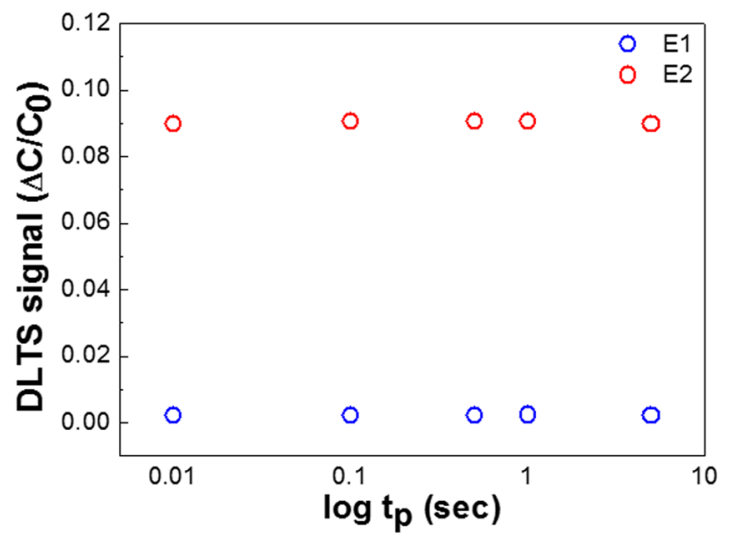

Figure 3. DLTS signals of E1 and E2 as a function of filling pulse time $t_{\mathrm{p}}$.

(a)

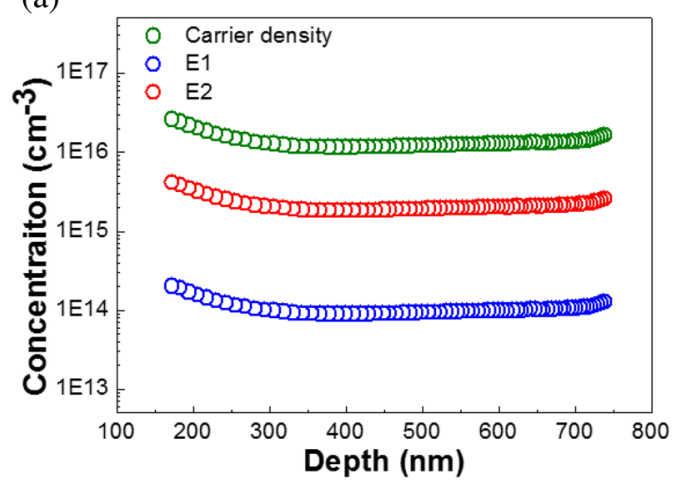

(b)

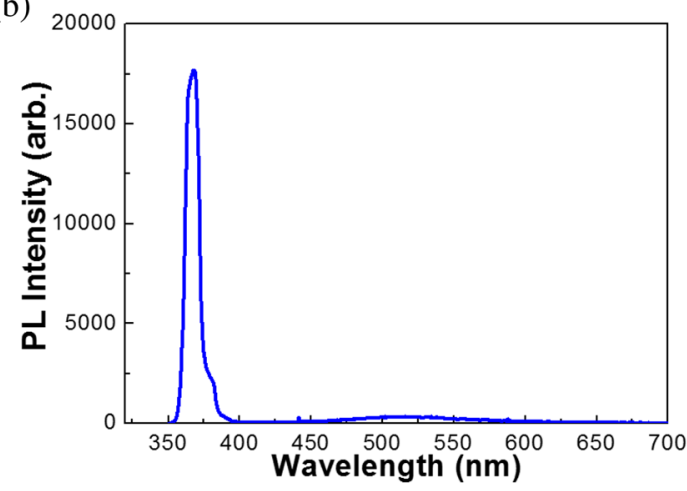

Figure 4. (a) Concentrations of carrier density and trap densities of E1 and E2 as a function of the distance from the surface of the Si-based FS-GaN. (b) Room temperature PL spectrum of the Si-based FS-GaN.

appeared near $3.375 \mathrm{eV}^{32}$. The full width at half maximum (FWHM) of the edge peak was approximately $8.5 \mathrm{~nm}$. The PL peaks of the samples exhibited a red-shift of $96 \mathrm{meV}$ with respect to those of completely relaxed bulk GaN $(3.471 \mathrm{eV})$, indicating the presence of tensile stress in the FS-GaN ${ }^{33,34}$. This is attributed to the crystal quality difference between the Ga- and $\mathrm{N}$-face surfaces of the FS- $\mathrm{GaN}^{35}$. It is essential to note that the slight plateau around wavelength of $514.5 \mathrm{~nm}$ can be clearly observed. Indeed, the typical yellow bands are positioned at $2.1-2.3 \mathrm{eV}$. However, broad band with maxima at $514.5 \mathrm{~nm}$ in the Fig. 4 can simulate green band rather than yellow luminescence. Even if a number of the exploration for the green emission from the GaN materials were implemented, its origin has not been clearly discovered up to now ${ }^{36,37}$. Indeed, some literatures stated that it may be associated with $\mathrm{V}_{\mathrm{N}}$, and $\mathrm{V}_{\mathrm{Ga}}{ }^{38}$. Based on PL and DLTS analyses, the Si-based FS-GaN may involve high nitrogen concentration. Note that the two defects are related to $\mathrm{V}_{\mathrm{Ga}}, \mathrm{V}_{\mathrm{N}}, \mathrm{V}_{\mathrm{Ga}}-\mathrm{V}_{\mathrm{N}}$ pair, $\mathrm{N}_{\mathrm{I}}$-related defects, indicating high nitrogen fraction in the GaN films. Lymperakis et al. also stressed that the high strain field of the dislocation in the GaN layers can induce a metal-like structure of the Ga-Ga bonds ${ }^{39}$. Moreover, they addressed that these strain-induced metallic bond states can be observed in the GaN crystals grown by MOCVD or HVPE, which contain the abundant stress and nitrogen concentration in the layer ${ }^{39}$. It is noticeable that Si-based FS-GaN underwent a huge tensile strain during the HVPE growth ${ }^{16}$. Furthermore, we also observed metallic Ga-Ga bonding in GaN template on a Si substrate, which underwent high tensile strain during the growth stage ${ }^{40}$. However, we cannot observe any evidence on the electronic deep level states related to the metallic Ga bonding in Si-based FS-GaN. Consequently, the characteristics of the electronic states of FS-GaN crystal extracted from a Si substrate is comparable to that of conventional freestanding GaN layers, despite of using a Si material. This indicates that Si-based FS-GaN can be a promising material to achieve the high performance opto-electronic devices with large scalability and low cost. To verify the energy states of the metallic bonds, electron energy loss spectroscopy (EELS) combined with scanning transmission electron microscopy (STEM) analysis is under investigation.

\section{Conclusion}

The electronic states of the deep levels in Si-based FS-GaN were investigated using DLTS analysis. Two deep trap levels were observed at $\mathrm{E}_{\mathrm{c}}-0.24 \mathrm{eV}$ and $\mathrm{E}_{\mathrm{c}}-1.06 \mathrm{eV}$ with capture cross sections of $1.65 \times 10^{-17} \mathrm{~cm}^{-2}$ and $1.76 \times 10^{-14} \mathrm{~cm}^{-2}$ and trap densities of $1.07 \times 10^{14} \mathrm{~cm}^{3}$ and $1.19 \times 1015 \mathrm{~cm}^{3}$, respectively. The DLTS signals as a function of filling pulse time and trap density depth profiles prove that the two deep levels are involved in noninteracting point defects. The PL measurement revealed that the green emission was observed, inherited from 
unknown sources. Despite of originating from a Si substrate, The properties of the electronic deep level states of Si-based FS-GaN is comparable to those of conventional one. We believe that the Si-based FS-GaN exhibits the desirable characteristics for large-scale opto-electronic applications with low production costs.

\section{Methods}

The fingerprints of electronic deep centers including the activation energy $\left(\mathrm{E}_{\mathrm{a}}\right)$ and capture cross section $\left(\sigma_{\mathrm{n}}\right)$ of trap states, extracted from Arrhenius plots, can be evaluated as follows ${ }^{41}$ :

$$
\operatorname{In}\left(\frac{\mathrm{e}_{\mathrm{n}}}{\mathrm{T}^{2}}\right)=\operatorname{In}\left(\sqrt{6} \pi 1.5 k^{2} \mathrm{~m}_{\mathrm{n}}^{*} \sigma_{\mathrm{n}} / \mathrm{h}^{3}\right)+\left(-\Delta E_{\mathrm{a}} / 1000 \mathrm{k}\right) 1000 / \mathrm{T}
$$

where $e_{n}, T, k, m^{*}$, and $h$ indicate the emission rate, the absolute temperature, Boltzmann's constant, the effective mass of the carrier, and Plank's constant, respectively. The trap parameters are presented in Table 1.

Furthermore, the trap density can be determined as a following equation ${ }^{42}$ :

$$
\mathrm{N}_{\mathrm{t}}=2 \mathrm{~N}_{\mathrm{d}} \mathrm{F}^{-1} \Delta C / \mathrm{C}
$$

where $\mathrm{N}_{\mathrm{d}}, \Delta \mathrm{C}$, and $\mathrm{F}$ indicate the donor concentration, the capacitance change during relaxation, and the spectrometer function of 3.5 used in this work, respectively.

\section{References}

1. Chung, K. et al. Growth and characterizations of GaN micro-rods on graphene films for flexible light emitting diodes. Apl Materials 2, 092512 (2014).

2. Shih, H.-Y. et al. Ultralow threading dislocation density in GaN epilayer on near-strain-free GaN compliant buffer layer and its applications in hetero-epitaxial LEDs. Scientific reports 5, 13671 (2015)

3. Suihkonen, S., Pimputkar, S., Speck, J. S. \& Nakamura, S. Infrared absorption of hydrogen-related defects in ammonothermal GaN. Applied Physics Letters 108, 202105 (2016).

4. Ma, H. et al. GaN crystals prepared through solid-state metathesis reaction from $\mathrm{NaGaO} 2$ and $\mathrm{BN}$ under high pressure and high temperature. Journal of Alloys and Compounds 509, L124-L127 (2011).

5. Takeuchi, S. et al. Dislocation confinement in the growth of $\mathrm{Na}$ flux $\mathrm{GaN}$ on metalorganic chemical vapor deposition-GaN. Journal of Applied Physics 118, 245306 (2015).

6. Jachalke, S. et al. The pyroelectric coefficient of free standing GaN grown by HVPE. Applied Physics Letters 109, 142906 (2016).

7. Cich, M. J. et al. Bulk GaN based violet light-emitting diodes with high efficiency at very high current density. Applied Physics Letters 101, 223509 (2012).

8. Kelly, M. K. et al. Large free-standing GaN substrates by hydride vapor phase epitaxy and laser-induced liftoff. Japanese journal of applied physics 38, L217 (1999).

9. Armitage, R. et al. Lattice-matched HfN buffer layers for epitaxy of GaN on Si. Applied Physics Letters 81, 1450-1452 (2002).

10. Abstracts of Papers, S. A. N. M., San Diego, C. A, United States, April 1-5, 2001 Able, Andreas, Wegscheider, W., Engl, K. \& Zweck, J. Growth of crack-free GaN on $\mathrm{Si}\left(\begin{array}{lll}1 & 1 & 1\end{array}\right)$ with graded AlGaN buffer layers. Journal of Crystal Growth 276, 415-418 (2005).

11. Arslan, E., Ozturk, M. K., Teke, A., Ozcelik, S. \& Ozbay, E. Buffer optimization for crack-free GaN epitaxial layers grown on Si (1 11 1) substrate by MOCVD. Journal of Physics D: Applied Physics 41, 155317 (2008).

12. Yang, T. H. et al. Growth of free-standing GaN layer on Si (1 11 1) substrate. Journal of Crystal Growth 311, 1997-2001 (2009).

13. Bessolov, V. et al. Semipolar AlN and GaN on Si (100): HVPE technology and layer properties. Journal of Crystal Growth 457, 202-206 (2017).

14. Lee, M., Yang, M., Song, K. M. \& Park, S. InGaN/GaN Blue Light Emitting Diodes Using Freestanding GaN Extracted from a Si Substrate. ACS Photonics (2018).

15. Lee, M. et al. A Novel Growth Method of Freestanding GaN Using In situ Removal of Si Substrate in Hydride Vapor Phase Epitaxy. Applied Physics Express 6, 125502 (2013).

16. Lee, M., Mikulik, D., Yang, M. \& Park, S. The investigation of stress in freestanding GaN crystals grown from Si substrates by HVPE. Scientific Reports 7, 8587 (2017).

17. Lee, M., Vu, T., Lee, K., Kim, E. \& Park, S. Electronic Transport Mechanism for Schottky Diodes Formed by Au/HVPE a-Plane GaN Templates Grown via In Situ GaN Nanodot Formation. Nanomaterials 8, 397 (2018).

18. Miller, E., Yu, E., Waltereit, P. \& Speck, J. Analysis of reverse-bias leakage current mechanisms in GaN grown by molecular-beam epitaxy. Applied physics letters 84, 535-537 (2004).

19. Duc, T. T., Pozina, G., Janzén, E. \& Hemmingsson, C. Investigation of deep levels in bulk GaN material grown by halide vapor phase epitaxy. Journal of Applied Physics 114, 153702 (2013).

20. Fang, Z. et al. Characteristics of deep traps in freestanding GaN. MRS Online Proceedings Library Archive 693 (2001).

21. Fang, Z.-Q. et al. Deep centers in a free-standing GaN layer. Applied Physics Letters 78, 2178-2180 (2001).

22. Lee, I.-H. et al. Electrical properties and radiation detector performance of free-standing bulk n-GaN. Journal of Vacuum Science \& Technology B, Nanotechnology and Microelectronics: Materials, Processing, Measurement, and Phenomena 30, 021205 (2012).

23. Polyakov, A. et al. Deep traps determining the non-radiative lifetime and defect band yellow luminescence in $\mathrm{n}-\mathrm{GaN}$. Journal of Alloys and Compounds 686, 1044-1052 (2016).

24. Cho, H., Khan, F., Adesida, I., Fang, Z. \& Look, D. C. Deep level characteristics in n-GaN with inductively coupled plasma damage. Journal of Physics D: Applied Physics 41, 155314 (2008).

25. Cho, H., Kim, C. \& Hong, C.-H. Electron capture behaviors of deep level traps in unintentionally doped and intentionally doped n-type GaN. Journal of applied physics 94, 1485-1489 (2003).

26. Chen, S. et al. As-grown deep-level defects in $\mathrm{n}-\mathrm{GaN}$ grown by metal-organic chemical vapor deposition on freestanding GaN. Journal of Applied Physics 112, 053513 (2012).

27. Choi, K. J., Jang, H. W. \& Lee, J.-L. Observation of inductively coupled-plasma-induced damage on n-type GaN using deep-level transient spectroscopy. Applied physics letters 82, 1233-1235 (2003).

28. Duc, T. T. et al. Electronic properties of defects in high-fluence electron-irradiated bulk GaN. physica status solidi (b) 253, 521-526 (2016).

29. Fang, Z., Look, D. C. \& Polenta, L. Dislocation-related electron capture behaviour of traps in n-type GaN. Journal of Physics: Condensed Matter 14, 13061 (2002).

30. Hierro, A. et al. Capture Kinetics of Electron Traps in MBE-Grown n-GaN. physica status solidi (b) 228, 309-313 (2001).

31. DeLucca, J., Mohney, S., Auret, F. \& Goodman, S. Pt Schottky contacts to $\mathrm{n}-\mathrm{GaN}$ formed by electrodeposition and physical vapor deposition. Journal of Applied Physics 88, 2593-2600 (2000). 
32. Chichibu, S., Azuhata, T., Sota, T. \& Nakamura, S. Excitonic emissions from hexagonal GaN epitaxial layers. Journal of applied physics 79, 2784-2786 (1996).

33. Kasic, A. et al. Highly homogeneous bulk-like 2" GaN grown by HVPE on MOCVD-GaN template. Journal of Crystal Growth 275 , e387-e393 (2005)

34. Gogova, D., Talik, E., Ivanov, I. G. \& Monemar, B. Large-area free-standing GaN substrate grown by hydride vapor phase epitaxy on epitaxial lateral overgrown GaN template. Physica B: Condensed Matter 371, 133-139 (2006).

35. Park, S. S., Park, I.-W. \& Choh, S. H. Free-standing GaN substrates by hydride vapor phase epitaxy. Japanese Journal of Applied Physics 39, L1141 (2000).

36. Reshchikov, M. A., Demchenko, D., Usikov, A., Helava, H. \& Makarov, Y. Carbon defects as sources of the green and yellow luminescence bands in undoped GaN. Physical Review B 90, 235203 (2014).

37. Reshchikov, M., McNamara, J., Helava, H., Usikov, A. \& Makarov, Y. Two yellow luminescence bands in undoped GaN. Scientific reports 8,8091 (2018).

38. Kojima, K. et al. Nitrogen vacancies as a common element of the green luminescence and nonradiative recombination centers in Mg-implanted GaN layers formed on a GaN substrate. Applied Physics Express 10, 061002 (2017).

39. Lymperakis, L., Neugebauer, J., Albrecht, M., Remmele, T. \& Strunk, H. Strain induced deep electronic states around threading dislocations in GaN. Physical review letters 93, 196401 (2004).

40. Lee, M. et al. Partial edge dislocations comprised of metallic Ga bonds in heteroepitaxial GaN. Nano letters 18, 4866-4870 (2018).

41. Kumar, A., Verma, S., Kumar, P., Kandasami, A. \& Kanjilal, D. K. Identification of Swift Heavy Ion Induced Defects in Pt/n-GaN Schottky Diodes by in-situ Deep Level Transient Spectroscopy. Semiconductor Science and Technology (2018).

42. Armstrong, A. et al. Impact of deep levels on the electrical conductivity and luminescence of gallium nitride codoped with carbon and silicon. Journal of Applied physics 98, 053704 (2005).

\section{Acknowledgements}

This research was supported by the Basic Science Research Program through the National Research Foundation of Korea (NRF), funded by the Ministry of Education (Nos 2018R1D1A1B07042909, 2018R1D1A1B07042909 and 2016R1A6A1A03012069), the Korea Institute of Energy Technology Evaluation and Planning (KETEP), the Ministry of Trade, Industry and Energy (MOTIE) of the Republic of Korea (No. 20163030013380), and the Korea Basic Science Institute research Grant No. C38711.

\section{Author Contributions}

M. Lee conceived the idea, performed the GaN epitaxial growth and decipher the DLTS measurement. C.W. Ahn and T.K.O. Vu performed fabrication and characterization of Schottky diodes for DLTS measurements. H.U. Lee conducted I-V characterization and its interpretation. E.K. Kim and S. Park designed the DLTS analysis and supervised the project. All authors discussed and reviewed the manuscript.

\section{Additional Information}

Competing Interests: The authors declare no competing interests.

Publisher's note: Springer Nature remains neutral with regard to jurisdictional claims in published maps and institutional affiliations.

(c) (1) Open Access This article is licensed under a Creative Commons Attribution 4.0 International License, which permits use, sharing, adaptation, distribution and reproduction in any medium or format, as long as you give appropriate credit to the original author(s) and the source, provide a link to the Creative Commons license, and indicate if changes were made. The images or other third party material in this article are included in the article's Creative Commons license, unless indicated otherwise in a credit line to the material. If material is not included in the article's Creative Commons license and your intended use is not permitted by statutory regulation or exceeds the permitted use, you will need to obtain permission directly from the copyright holder. To view a copy of this license, visit http://creativecommons.org/licenses/by/4.0/.

(c) The Author(s) 2019 